\title{
Synthesis of divinyl protochlorophyllide
}

\section{Enzymological properties of the Mg-protoporphyrin IX monomethyl ester oxidative cyclase system}

\author{
Caroline J. WALKER, ${ }^{*}$ Paul A. CASTELFRANCO $\dagger$ and Barry J. WHYTE \\ Department of Botany, University of California at Davis, Davis, CA 95616, U.S.A.
}

\begin{abstract}
The resolution and reconstitution of the Mg-protoporphyrin IX monomethyl ester oxidative cyclase system into a supernatant and a pellet fraction was accomplished by a procedure involving salt treatment followed by osmotic shock. Recombination of pellet and supernatant fractions was required for cyclase activity. This restoration effect could be demonstrated using either Mg-protoporphyrin IX or Mg-protoporphyrin IX monomethyl ester as the cyclase substrate in the presence or absence of $S$-adenosylmethionine. Pretreatment of the pellet fraction with either 8-hydroxyquinoline or desferal mesylate inhibited cyclase activity, indicating that there is a heavy-metal-ion requirement in this fraction. The cyclase supernatant protein(s) was not internalized by Sephadex G-50 and did not bind to Blue Sepharose, suggesting that it has a molecular mass of over $30 \mathrm{kDa}$ and that it does not bind the cofactor NADPH. The cyclase supernatant protein did bind to $\mathrm{MgProtoMe}_{2}$-bound Sepharose and could be eluted by raising the $\mathrm{pH}$ to 9.7 in the presence of $4 \mathrm{~mm}$-n-octyl glucoside. The $\mathrm{pH}$ optimum of the cyclase was 9.0 . About a 40 -fold purification of the cyclase supernatant protein was achieved by a combination of $\left(\mathrm{NH}_{4}\right)_{2} \mathrm{SO}_{4}$ fractionation and phenyl-Sepharose chromatography.
\end{abstract}

\section{INTRODUCTION}

The Mg-protoporphyrin IX monomethyl ester (MgProtoMe) oxidative cyclase system (or cyclase) catalyses the conversion of MgProtoMe to protochlorophyllide (Pchlide) in the chlorophyll (Chl) synthesis pathway. Granick (1950) proposed that isocyclicring synthesis involved the $\beta$-oxidation of the 6-methylpropionate side chain of MgProtoMe to a methyl- $\beta$-oxopropionate group. The modification of the 6-methylpropionate side chain was thought to go through 6-acrylate and 6- $\beta$-hydroxypropionate intermediates by analogy to the $\beta$-oxidation of fatty acids.

Recent work in this laboratory has both confirmed and modified this scheme. The chemically synthesized 6-acrylate derivative of MgProtoMe proved to be an inactive substrate for the cyclase (Walker et al., 1988), whereas both the $6-\beta$ hydroxypropionate and 6- $\beta$-oxopropionate derivatives could be converted into Pchlide (Wong \& Castelfranco 1984, 1985; Wong et al., 1985; Walker et al., 1988). These findings suggested a hydroxylation reaction involving atmospheric oxygen. This model was subsequently confirmed by the observation of the direct incorporation of molecular oxygen into the isocyclic ring (Walker et al., 1989).

Although our understanding of the mechanism of the cyclase has improved, there is still little information on the individual enzymes. Since several intermediates are involved in the reaction, the cyclase must be a collection or a complex of several enzymes. Previous work has shown that it is possible to resolve the cyclase into membrane-bound and soluble fractions by the sonication of intact plastids and ultracentrifugation (Wong \& Castelfranco, 1984). Recombination of the pellet and supernatant fractions was required to restore cyclase activity. Neither fraction alone was sufficient to catalyse the conversion of chemically synthesized intermediates to Pchlide, for which the two cofactors, NADPH and $\mathrm{O}_{2}$, were likewise required (Wong et al., 1985). The product of the cyclization reaction in vitro was shown to be divinylPchlide (2,4-divinylpheoporphyrin $a_{5}$ ) (Chereskin et al., 1983).

In the present study we have continued to investigate the enzymology of the cyclase using a reconstituted system derived from salt-shocked and osmotically lysed, rather than sonicated, plastids. The membrane-bound component exhibits a heavymetal requirement, which agrees with the results of previous studies carried out with whole plastids (Chereskin \& Castelfranco, 1982; Nasrulhaq-Boyce et al., 1987). Evidence is presented that the soluble fraction binds the porphyrin, but not the other substrate for the cyclase, NADPH. Partial purification of the supernatant protein is described.

\section{MATERIALS AND METHODS}

\section{Materials}

General. Hepes, Tes, NADPH (tetrasodium salt, chemically reduced), $S$-adenosylmethionine (SAM ; chloride salt), cysteine hydrochloride, EDTA, sorbitol, SDS, Trizma, glycine, bisacrylamide, $\left(\mathrm{NH}_{4}\right)_{2} \mathrm{SO}_{4}$, Coomassie Brilliant Blue G-250, 2mercaptoethanol, ammonium persulphate and Sepharose 4B (CNBr-activated) were purchased from Sigma. $\mathrm{NaCl}$, trichloroacetic acid and all organic solvents were obtained from Fisher. Spectrapor dialysis tubing was obtained from American Scientific Products; $N N N^{\prime} N^{\prime}$-tetramethylethylenediamine (TEMED) and acrylamide were purchased from Bio- $\mathrm{Rad} . \mathrm{MgCl}_{2}$ was obtained from Mallinckrodt. Centricon 10 microconcentrators were purchased from Amicon. BSA (fraction V, fatty-acid-poor) was from Calbiochem. Sephadex G-50 was purchased from Pharmacia. Cucumber (Cucumis sativus L., cv. Beit Alpha) seeds were a gift from Harris Moran Seeds, Salinas, CA, U.S.A. Desferal mesylate was a gift from CIBA Pharmaceutical Co., Summit, NJ 07901, U.S.A.

Abbreviations used: Chl, chlorophyll; CSP, cyclase supernatant protein(s); DMSO, dimethyl sulphoxide; MgProto, Mg-protoporphyrin IX; MgProtoMe, Mg-protoporphyrin IX monomethyl ester; MgProtoMe ${ }_{2}$, Mg-protoporphyrin IX dimethyl ester; ME, MgProtoMe esterase(s); MT, MgProto methyltransferase (EC 2.1.1.11); PAR, photosynthetically active radiation; Pchlide, protochlorophyllide (this abbreviation is used generically to denote the product of the 'in vitro' cyclization reaction without regard to the oxidation state of the 2 and 4 substituents); SAM, $S$-adenosylmethionine; TEMED, $N N N^{\prime} N^{\prime}$-tetramethylethylenediamine.

* Present address: Department of Biological Sciences, Clemson University, Clemson, SC 29634-1903, U.S.A.

$\dagger$ To whom correspondence should be addressed. 
Plant tissue. Cucumber seeds were germinated in the dark at $30^{\circ} \mathrm{C}$ for 5-6 days (Hardy et al., 1970). Etiolated seedlings were exposed to white light $\left(60-80 \mu \mathrm{E} \cdot \mathrm{m}^{-2} \cdot \mathrm{s}^{-1}\right.$ PAR at $\left.30^{\circ} \mathrm{C}\right)$ for $20 \mathrm{~h}$.

Mg-protoporphyrin IX (MgProto). This was prepared by alkaline hydrolysis of Mg-protoporphyrin IX dimethyl ester $\left(\mathrm{MgProtoMe}_{2}\right)($ Chereskin \& Castelfranco, 1982); the latter was obtained from Porphyrin Products, Logan, UT 84321, U.S.A. MgProtoMe was made biologically as described previously (Chereskin et al., 1982). All porphyrin substrates were dissolved in dimethyl sulphoxide (DMSO) at a concentration of $500 \mu \mathrm{M}$ before addition to cyclase assays.

\section{Methods}

Plastid isolation and resolution of the cyclase. Developing chloroplasts were isolated from greening cucumber cytoledons as described by Fuesler et al. (1984), except that 'pellet 2' was resuspended in $5 \mathrm{ml}$ (per pellet) of Buffer A ( $0.5 \mathrm{M}$-sorbitol/ $1 \mathrm{~mm} \mathrm{MgCl} / 1 \mathrm{~mm}$-EDTA/20 mM-Tes/10 mM-Hepes, pH 7.7). The pellet suspension was then illuminated for $5 \mathrm{~min}$ at $0{ }^{\circ} \mathrm{C}$ to remove remaining traces of Pchlide (Chereskin et al., 1982) before dilution with $35 \mathrm{ml}$ of Buffer $\mathrm{C}(0.2 \mathrm{M}-\mathrm{NaCl} / 1 \mathrm{~mm}-$ $\mathrm{MgCl}_{2} / 1 \mathrm{~mm}$-EDTA/20 mM-Tes/10 mm-Hepes, $\mathrm{pH} 7.7$ ), which causes the plastids to loose some integrity (Chereskin et al., 1982). After $10 \mathrm{~min}$ at $0^{\circ} \mathrm{C}$ the suspension was centrifuged at $500 \mathrm{~g}$ for $10 \mathrm{~min}$. The supernatant was discarded and the pellet vigorously resuspended in $3.4 \mathrm{ml}$ of hypo-osmotic Buffer $B$ (10 mu-Hepes/10 mu-Tes/1 mM-EDTA/1 mm- $\mathrm{MgCl}_{2}, \mathrm{pH}$ 7.7) and lysed for $5 \mathrm{~min}$ at $0{ }^{\circ} \mathrm{C}$, then centrifuged at $12000 \mathrm{~g}$ for $10 \mathrm{~min}$. The supernatant was kept and the pellet washed by resuspension in $35 \mathrm{ml}$ of Buffer B and centrifugation at $12000 \mathrm{~g}$ for $10 \mathrm{~min}$. Recombination of the pellet and supernatant was necessary to restore cyclase activity.

$\left(\mathrm{NH}_{4}\right)_{2} \mathrm{SO}_{4}$ precipitation and dialysis. In some experiments the supernatant was adjusted to $60 \%$-satd. $\left(\mathrm{NH}_{4}\right)_{2} \mathrm{SO}_{4}$ and, after $10 \mathrm{~min}$ at $0{ }^{\circ} \mathrm{C}$, centrifuged at $12000 \mathrm{~g}$ for $10 \mathrm{~min}$. The pellet was discarded and the supernatant was adjusted to $85 \%\left(\mathrm{NH}_{4}\right)_{2} \mathrm{SO}_{4}$ saturation. After $30 \mathrm{~min}$ at $0{ }^{\circ} \mathrm{C}$ the precipitated proteins were pelleted by centrifugation at $12000 \mathrm{~g}$ for $10 \mathrm{~min}$. Pellets were resuspended in a minimal volume of Buffer $B$ and dialysed against $500 \mathrm{ml}$ of the same buffer for $1 \mathrm{~h}$ at $4^{\circ} \mathrm{C}$. Saturated $\left(\mathrm{NH}_{4}\right)_{2} \mathrm{SO}_{4}$ solutions were adjusted to $\mathrm{pH} 7.7$ with concentrated aq. $\mathrm{NH}_{3}$ before use.

Column chromatography. Typically, chromatography was carried out with 1-2 ml of gel in $0.8 \mathrm{~cm}$-diameter columns. Flow was by gravity (rates were approx. $1 \mathrm{ml} / \mathrm{min}$ ), and samples and buffers were applied manually. Fractions $(2-3 \mathrm{ml})$ were collected on ice and concentrated to about $50 \mu 1$ by using microconcentrators. If $\left(\mathrm{NH}_{4}\right)_{2} \mathrm{SO}_{4}$ was included in the running buffers, fractions were dialysed (as described above) before concentration.

Preparation of $\mathrm{MgProtoMe}_{2}$ affinity column. $\mathrm{CNBr}$-activated Sepharose 4B was coupled to $\mathrm{MgProtoMe}_{2}$ by the method of Richards et al. (1987). An absorption spectrum of the gel in glycerol/methanol $(1: 1, \mathrm{v} / \mathrm{v})$ showed peaks at 557 and $595 \mathrm{~nm}$.

Incubation conditions. Routine incubations with the reconstituted system were carried out in a total volume of $1 \mathrm{ml}$ in Buffer B. Substrates and cofactors were $10 \mu \mathrm{M}$-MgProto or MgProtoMe, $1 \mathrm{mM}-\mathrm{SAM}$ and $5 \mathrm{mM}-\mathrm{NADPH}$; the samples were incubated in darkness to prevent phototransformation or photodestruction of newly formed Pchlide. Incubations were carried out at $30^{\circ} \mathrm{C}$ for $1 \mathrm{~h}$ in a metabolic shaker at $60-65$ double strokes/min and terminated by freezing at $-15^{\circ} \mathrm{C}$.

Pchlide extraction and determination. Pchlide formed during the incubation was extracted into diethyl ether as described by Castelfranco et al. (1979) and was determined by using a Perkin-Elmer MP 44-A fluorescence spectrophotometer as described by Chereskin et al. (1982).
Protein determinations. Protein was determined by the method of Bradford (1976), with BSA as a standard.

H.p.l.c. Pigments were separated by the h.p.l.c. system previously described by Fuesler et al. (1982).

Quantitative expression of the cyclase assay. Cyclase activity is expressed in pmol of Pchlide/h per $\mathrm{mg}$ of protein. The legend to each table indicates whether this activity is expressed with respect to pellet, cyclase supernatant protein (CSP) or total protein. All other departures are likewise stated. Incubations were carried out for $1 \mathrm{~h}$ in order to increase the yield of product. It was established that cyclase activity was directly proportional to the amount of plastid protein added. Hence all assay values are comparable, even though Pchlide formation is not linear with time (Walker et al., 1988).

SDS/PAGE. SDS/PAGE analysis of protein fractions were carried out by the method of Laemmli (1970). All analyses were done using $12 \%-(\mathrm{w} / \mathrm{v})$-acrylamide separating gels. Standard proteins ( $\alpha$-lactalbumin, $14.2 \mathrm{kDa}$; trypsin inhibitor, $21.1 \mathrm{kDa}$; carbonic anhydrase, $29.0 \mathrm{kDa}$; glyceraldehyde-3-phosphate dehydrogenase, $36.0 \mathrm{kDa}$; egg albumin, $45.0 \mathrm{kDa}$; bovine serum albumin, $66.0 \mathrm{kDa}$ ) were co-electrophoresed for molecular-mass estimations.

\section{RESULTS}

Comparison of sonication and osmotic-shock methods for the resolution of the cyclase.

Table 1 compares typical reconstitution experiments according to the method described by Wong \& Castelfranco (1984) and the osmotic-lysis method used in all the experiments reported here. In both cases, the pellet or supernatant alone had little cyclase activity, but recombination of these fractions restored considerable activity. In these experiments the small activity measured in the 'pellet alone' controls was subtracted from the activity of the other samples. Pellet fractions could be stored in Buffer $\mathrm{B} /$ glycerol $(1: 1, \mathrm{v} / \mathrm{v})$ at $-80^{\circ} \mathrm{C}$; supernatant fractions were stable at $-80^{\circ} \mathrm{C}$ in Buffer $\mathrm{B}$.

\section{Table 1. Resolution and reconstitution of the cyclase}

Resolution and reconstitution of the cyclase was performed as described by Wong \& Castelfranco (1984) (Method 1) or as described under 'Methods' (Method 2), except that the pellet was not washed, in order to allow direct comparison of the two techniques. Component concentrations were: MgProto, $10 \mu \mathrm{M}$; NADPH, $5 \mathrm{~mm}$; SAM, 1 mM. The protein values used to calculate the cyclase activities represent the total protein in each incubation. Other incubation conditions were as described under 'Methods'. In all Tables, where shown, entries represent the mean and range of duplicates, and the term 'Pchlide' is used for the 'in vitro' cyclization product without regard to the oxidation state of the 2 and 4 substituents (Fischer formula).

\begin{tabular}{lcr}
\hline & \multicolumn{2}{c}{ Cyclase activity } \\
\cline { 2 - 3 } & $\begin{array}{c}\text { (pmol of Pchlide/ } \\
\text { M per mg of protein) }\end{array}$ & $\begin{array}{c}\text { (\% of that } \\
\text { intact plas }\end{array}$ \\
\hline Method and fraction & & 100 \\
Method 1 : sonication & $350 \pm 11$ & 6 \\
Intact plastids & 21 & 7 \\
Pellet & 25 & 40 \\
Supernatant & $140 \pm 10$ & 100 \\
Pellet + supernatant & $208 \pm 12$ & 12 \\
Method 2: osmotic lysis & $24 \pm 9$ & 0 \\
Intact plastids & 0 & 52 \\
Pellet & $109 \pm 2$ & \\
Supernatant & & \\
Pellet + supernatant & &
\end{tabular}




\section{Table 2. Activity of the reconstituted cyclase with different substrates}

The reconstituted cyclase was tested in four separate experiments with four substrate conditions: $10 \mu \mathrm{M}$-MgProto or $10 \mu \mathrm{M}$ MgProtoMe, with or without $1 \mathrm{mM}-\mathrm{SAM}$. The protein values used to calculate the cyclase activities in lysed chloroplast preparations were for CSP only, but in the unresolved chloroplast controls they represented total protein. During the resolution the protein of the original chloroplast is divided up as follows: CSP, $36 \%$; pellet, $35 \%$; lost, $29 \%$. Washed pellets alone showed no activity. Other incubation conditions were as described in Table 1 and under 'Methods'.

\begin{tabular}{lcc}
\hline Substrates & $\begin{array}{c}\text { Enzyme } \\
\text { system }\end{array}$ & $\begin{array}{c}\text { Cyclase activity } \\
\text { (pmol of Pchlide/ } \\
\text { h per mg of protein) }\end{array}$ \\
\hline MgProto & CSP & 0 \\
CSP + pellet & $8 \pm 0$ \\
MgProto+SAM & Control & 38 \\
CSP & 0 \\
MgProtoMe & CSP + pellet & $61 \pm 1$ \\
& Control & 168 \\
MgProtoMe +SAM & CSP & 0 \\
& CSP+ pellet & $35 \pm 7$ \\
& Control & 63 \\
& CSP & 0 \\
& CSP + pellet & $111 \pm 9$ \\
& Control & 199 \\
\hline
\end{tabular}

\section{Comparison of MgProto and MgProtoMe as substrates for the} reconstituted cyclase system.

The reconstitution of the cyclase activity was tested using two Mg-porphyrin substrates, MgProto and MgProtoMe, with or without SAM (Table 2). In all cases the pellet and the supernatant alone had no activity, but the recombined samples had significant activity. The activities of the recombined samples are expressed on a CSP basis, but the activities of the chloroplasts before salt treatment (controls) are expressed in terms of total plastid protein. In order to compare the activity of the initial pellet with that of the reconstituted system, one must keep in mind that, during the resolution, $35 \%$ of the protein remains in the pellet, $36 \%$ ends up in the supernatant and $29 \%$ is lost.

The protein that was washed away in the salt treatment was recovered by $\left(\mathrm{NH}_{4}\right)_{2} \mathrm{SO}_{4}$ precipitation and dialysed against buffer.
This fraction was shown to contain no CSP activity and not to be inhibitory.

As expected, SAM strongly enhanced the cyclization of MgProto, but also stimulated the cyclization of MgProtoMe, because of the combined action of MgProtoMe esterase(s) (ME) and MgProto methyltransferase (MT) present in the chloroplasts (Chereskin et al., 1982).

After the spectrofluorimetric determination of the reaction product, the porphyrin mixtures were analysed by h.p.l.c. After the salt-osmotic-shock treatment the ME activity was found in the CSP, and MT activity in the pellet, since CSP alone was able to convert MgProtoMe into MgProto, and pellet alone was able to convert MgProto into MgProtoMe in the presence of SAM. Hardly any esterase activity was found in the pellet, or any transferase activity in the CSP (results not shown).

In the subsequent experiments described in this paper, $10 \mu \mathrm{M}$ MgProto and $1 \mathrm{~mm}-\mathrm{SAM}$ were used, endogenous MT activity being relied upon to produce the oxidative cyclization substrate, MgProtoMe, during the incubation. However, in the experiments on the $\mathrm{pH}$-dependence of the cyclase reaction and on the stability of CSP, MgProtoMe was substituted for MgProto in order to ensure that the $\mathrm{pH}$-dependence measured was, so far as possible, that of the cyclase itself.

The action of metal-ion chelators on the cyclase. Previous experiments have shown that the cyclase can be inhibited by metal-ion chelators (Nasrulhaq-Boyce et al., 1987). Table 3 shows the effect of 8-hydroxyquinoline and desferal mesylate on the pellet fraction of the cyclase. In this experiment pellet fractions were exposed to the chelator for $10 \mathrm{~min}$ at $0^{\circ} \mathrm{C}$, then washed twice with Buffer $B$ to remove the chelator before recombination with untreated supernatant fraction. With both chelators, cyclase activity was partially inhibited; 8-hydroxyquinoline (10 mM was required for $89 \%$ inhibition) was a stronger inhibitor than desferal mesylate $(90 \mathrm{~mm}$ was required for $82 \%$ inhibition). To ascertain whether chelator remained in the pellet after the buffer wash, the chelator-treated and -untreated pellets were combined in a 1:1 ratio and incubated with the supernatant fraction. It was expected that any chelator trapped in the treated pellet would be free to inhibit the untreated pellet during the incubation. Table 3 shows that, with 8-hyroxyquinoline, the activity of the mixture was $38 \%$ of the untreated control, whereas the predicted activity was $55 \%$, suggesting that some chelator may have been retained by the treated pellet. In contrast, at both 10 and $90 \mathrm{~mm}$-desferal mesylate, the observed activities for the mixture were approximately those predicted. Cyclase

Table 3. Pretreatment of the pellet fraction with 8-hydroxyquinoline and desferal mesylate

Pellet fractions were exposed to the chelator concentration indicated at $0{ }^{\circ} \mathrm{C}$ for $10 \mathrm{~min}$. Chelator was removed by diluting $1 \mathrm{ml}$ of pellet suspension with $35 \mathrm{ml}$ of Buffer B, centrifuging at $12000 \mathrm{~g}$ for $10 \mathrm{~min}$, and finally washing the pellet once more in $35 \mathrm{ml}$ of Buffer B. 'Control' samples were treated identically, except for the omission of chelator. Treated and untreated pellets were recombined with their respective CSP that had been stored on ice; pellet recovery was assumed to be $100 \%$. In 'control + chelator-treated' samples, half the usual amount of pellet was taken from each sample. Values in parentheses are percentages of control values; emboldened values are the predicted activities for these samples. Totalprotein values were used to calculate cyclase activities. Substrates and incubations were as described in the legend to Table 1 .

Cyclase activity

(pmol of Pchlide/h per $\mathrm{mg}$ of protein)

\begin{tabular}{|c|c|c|c|}
\hline \multirow{2}{*}{$\begin{array}{r}\text { Chelator } \ldots \\
\text { Concn. (mM)... }\end{array}$} & \multirow{2}{*}{$\frac{8 \text {-Hydroxyquinoline }}{10}$} & \multicolumn{2}{|c|}{ Desferal mesylate } \\
\hline & & 90 & 10 \\
\hline $\begin{array}{l}\text { Control } \\
\text { Chelator-treated } \\
\text { Control }+ \\
\text { chelator-treated }(1: 1)\end{array}$ & $\begin{array}{l}57 \pm 5(100) \\
6 \pm 0(11) \\
23 \pm 3[38(55)]\end{array}$ & $\begin{array}{l}15 \pm 1(100) \\
3 \pm 0(18) \\
8 \pm 0[52(59)]\end{array}$ & $\begin{array}{l}19 \pm 1(100) \\
11 \pm 1(57) \\
16 \pm 1[85(79)]\end{array}$ \\
\hline
\end{tabular}


Table 4. Chromatography of the supernatant fraction on Sephadex G-50 and Blue Sepharose

Columns were prepared and used as described under 'Methods'. For substrates and incubation conditions, see the legend to Table 1 . In the bioassay, pellet protein for Sephadex G-50 was $1 \mathrm{mg} / \mathrm{ml}$, and for Blue Sepharose, $1.3 \mathrm{mg} / \mathrm{ml}$. Recovery of the supernatant protein from the columns was not determined; the amount of pellet was known to be saturating (see Fig. 4 and Table 5).

\begin{tabular}{lcc}
\hline & \multicolumn{2}{c}{$\begin{array}{c}\text { Cyclase activity } \\
\text { (pmol of Pchlide/h) }\end{array}$} \\
\cline { 2 - 3 } Column fraction & Sephadex G-50 & Blue Sepharose \\
\hline Loaded sample & 173 & 108 \\
Void volume & 0 & 0 \\
Unabsorbed/excluded & 160 & 116 \\
Absorbed/internalized & 0 & 0 \\
\hline
\end{tabular}

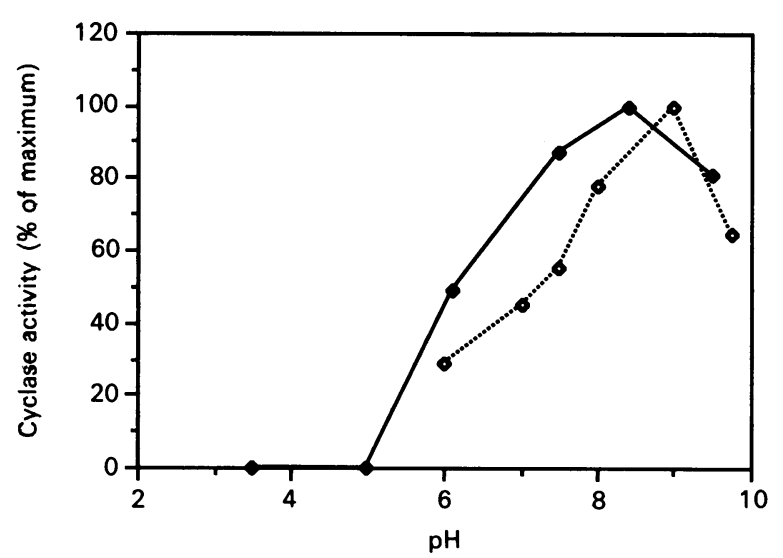

Fig. 1. pH-dependence of the cyclase reaction, and of CSP stability

For the pH-dependence of the cyclase reaction, the activity of the reconstituted system $(\diamond)$ was assayed in a buffer consisting of (20 mM each) citric acid, Mes, Hepes, Tricine and glycine, plus $1 \mathrm{~mm}$-EDTA and $1 \mathrm{~mm}-\mathrm{MgCl}_{2}$, adjusted to the appropriate $\mathrm{pH}$ with $\mathrm{KOH}$ or $\mathrm{HCl} ; 100 \%$ activity, taken at the $\mathrm{pH}$ optimum, was $167 \mathrm{pmol}$ of Pchlide/h per $\mathrm{mg}$ of protein. The stability of CSP ( $\bullet)$ was determined by incubating CSP at $0{ }^{\circ} \mathrm{C}$ for $2 \mathrm{~h}$ in the above buffers, followed by microconcentration to $50 \mu \mathrm{l}$ and resuspension in $500 \mu 1$ of Buffer B. CSP was bioassayed as described under 'Methods'. Substrates were $10 \mu \mathrm{M}-\mathrm{MgProtoMe}, 1 \mathrm{mM}-\mathrm{SAM}$ and $5 \mathrm{~mm}$-NADPH; $100 \%$ activity was $143 \mathrm{pmol}$ of Pchlide/h per $\mathrm{mg}$ of protein.

activities in this experiment were low because of the instability of the pellet under the treatment conditions. Complementary experiments pretreating the supernatant fraction with these metal-ion chelators were attempted, but in every case complete removal of the chelator by $\left(\mathrm{NH}_{4}\right)_{2} \mathrm{SO}_{4}$ precipitation and dialysis was not achieved.

Sephadex G-50 and Blue Sepharose chromatography. Attempts to solubilize the pellet fraction for further analysis were unsuccessful. However, it was found that the cyclase was stable to the detergent $\mathrm{n}$-octyl glucoside and was slightly stimulated by it at the lower concentrations (4-6 mM; results not shown). Subsequent experiments were therefore confined to the supernatant fraction.

Table 4 shows the behaviour of the supernatant fraction on two columns. Column fractions in these and all further experiments were bioassayed for cyclase activity by combining with pellet fraction (0.5-1.5 mg of protein per incubation) and incubating with the appropriate substrates as described under

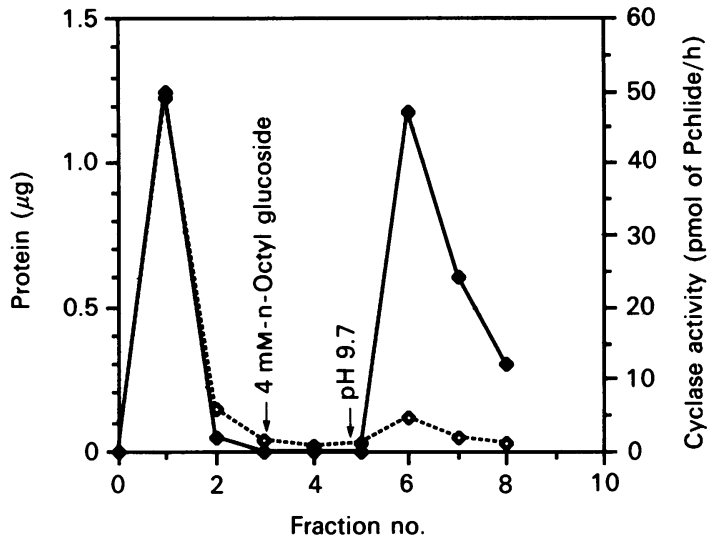

Fig. 2. Affinity chromatography of CSP on $\mathrm{MgProtoMe}_{2}$-bound Sepharose

Column chromatography and fraction bioassay was as described under 'Methods'. The protein $(2.8 \mathrm{mg})$ was loaded in $500 \mu \mathrm{l}$ of Buffer B and eluted at $\mathrm{pH} 9.7$ in the presence of $4 \mathrm{~mm}$-n-octyl glucoside. Cofactors and substrates were $10 \mu \mathrm{M}-\mathrm{MgProto}, 5 \mathrm{~mm}$ NADPH and $1 \mathrm{mM}-\mathrm{SAM}$. Pellet protein per bioassay was $1.0 \mathrm{mg}$. The addition of $4 \mathrm{~mm}$-n-octyl glucoside and the $\mathrm{pH}$ change to 9.7 are indicated in the Figure. $\diamond$, Protein; $\diamond$, cyclase activity.

'Methods' and in the Tables and Figure legends. The data in Table 4 show that CSP was not internalized by Sephadex G-50, indicating a molecular mass in excess of $30 \mathrm{kDa}$. On Sephadex G-100, CSP activity was lost (results not shown). Presumably, CSP was internalized, but was broken down with attempts to elute it from the column.

Two of the substrates for the cyclase are NADPH and MgProtoMe. It was therefore of interest to establish whether the supernatant fraction could bind either of these two compounds. Table 4 shows that CSP did not bind to Blue Sepharose, a gel which retains many nucleotide-binding enzymes (Heyns \& DeMoor, 1974). Washing with buffer containing $\mathrm{NaCl}(0.1-$ $0.3 \mathrm{M}$ ) failed to remove any CSP activity from the column.

pH responses of the cyclase. Fig. 1 shows the $\mathrm{pH}$-dependence of the stability of CSP and of the cyclase assay itself. After a $2 \mathrm{~h}$ preincubation at different $\mathrm{pH}$ values, all the CSP samples were adjusted to $\mathrm{pH} 7.7$ and tested as described under 'Methods'. CSP was stable at alkaline $\mathrm{pH}$, but was strongly inactivated below $\mathrm{pH}$ 7.0. The $\mathrm{pH}$ optimum of the cyclase assay was about pH 9.0.

$\mathrm{MgProtoMe}_{2}$ affinity chromatography. The ability of CSP to bind porphyrin was tested by affinity chromatography on a Sepharose column with bound $\mathrm{MgProtoMe}_{2}$ (see under 'Methods'). Elution from these types of columns often requires $\mathrm{pH}$ changes.

The chromatography of CSP on the $\mathrm{MgProtoMe}_{2}$ affinity column is presented in Fig. 2. The protein profile shows that most of the recovered protein $(88 \%)$ was not bound to this column. Some CSP was also present in this unabsorbed fraction (36\% of recovered activity). The addition of $4 \mathrm{mm-n-octyl}$ glucoside to the running buffer did not promote the dissociation of protein or of CSP from the column until the $\mathrm{pH}$ was raised to 9.7. A small amount of protein was eluted under these conditions that contained a significant amount of CSP $(64 \%$ of recovered activity). Raising the $\mathrm{pH}$ in the absence of n-octyl glucoside was not effective in eluting CSP (results not shown). The binding of CSP to the column could be increased to virtually $100 \%$ by loading in the presence of $4 \mathrm{~mm}$-n-octyl glucoside, but elution by raising the $\mathrm{pH}$ then did not occur (results not shown). Inclusion of $\mathrm{MgProtoMe}_{2}$ in elution buffers was ineffective, probably owing to the absorption of this porphyrin on to the column itself (results not shown). 


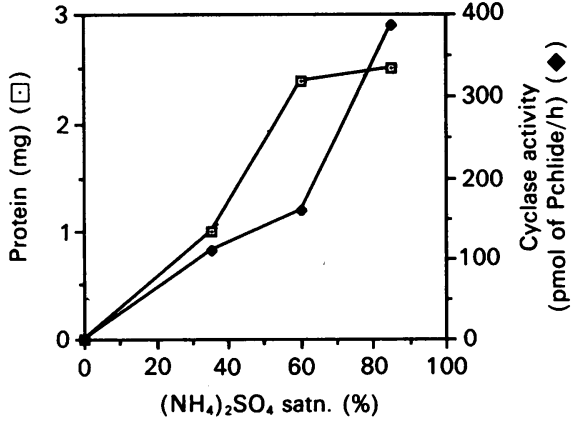

Fig. 3. $\left(\mathrm{NH}_{4}\right)_{2} \mathrm{SO}_{4}$ fractionation of the supernatant

The supernatant fraction was adjusted to either 35,60 or $85 \%$ $\left(\mathrm{NH}_{4}\right)_{2} \mathrm{SO}_{4}$ [by the addition of the appropriate amount of saturated $\left.\left(\mathrm{NH}_{4}\right)_{2} \mathrm{SO}_{4}\right]$ and, after $15 \mathrm{~min}$ at $0{ }^{\circ} \mathrm{C}$, insoluble proteins were collected by centrifugation at $12000 \mathrm{~g}$ for $10 \mathrm{~min}$. Remaining $\left(\mathrm{NH}_{4}\right)_{2} \mathrm{SO}_{4}$ in the precipitated proteins was removed by resuspension in Buffer $B$ and dialysis, as described under 'Methods'. Each sample was bioassayed for cyclase activity against $0.8 \mathrm{mg}$ of pellet protein The control sample was treated identically, with the exception that no $\left(\mathrm{NH}_{4}\right)_{2} \mathrm{SO}_{4}$ was added.

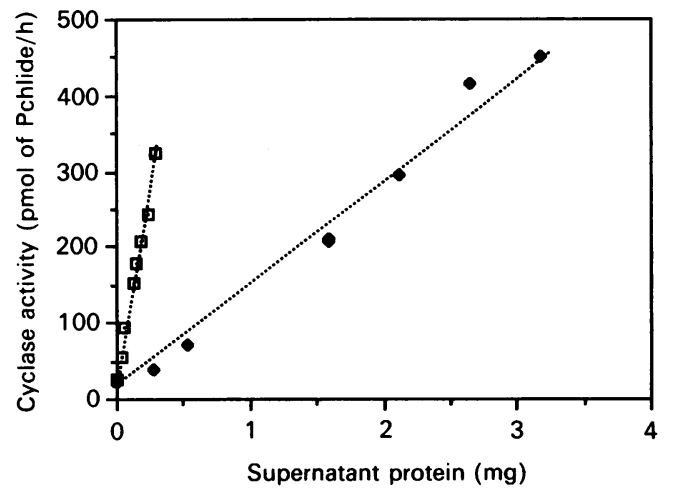

Fig. 4. Linearity of cyclase activity with supernatant protein in the bioassay procedure

Two preparations of CSP were bioassayed at different concentrations against a constant amount $(0.6 \mathrm{mg})$ of pellet protein: $\downarrow$, unfractionated supernatant; $\square, 60-85 \%$-satd.-( $\left(\mathrm{NH}_{4}\right)_{2} \mathrm{SO}_{4}$ fraction. Incubation and bioassay conditions were as described in the legend of Fig. 1.

$\left(\mathrm{NH}_{4}\right)_{2} \mathrm{SO}_{4}$ fractionation of CSP. Fig. 3 shows the protein and CSP activity precipitated from the supernatant at three different $\left(\mathrm{NH}_{4}\right)_{2} \mathrm{SO}_{4}$ concentrations. Although some CSP was precipitated by 35 and $60 \%$ saturation of $\left(\mathrm{NH}_{4}\right)_{2} \mathrm{SO}_{4}, 85 \%$-satd. $\left(\mathrm{NH}_{4}\right)_{2} \mathrm{SO}_{4}$ gave the greatest yield of CSP. However, the protein precipitated by $60 \%$-satd. $\left(\mathrm{NH}_{4}\right)_{2} \mathrm{SO}_{4}$ was comparable in amount with that precipitated at $85 \%$ (Fig. 3). Thus significant purification of CSP from the supernatant was achieved by removing those proteins which were insoluble at $60 \%$-satd. $\left(\mathrm{NH}_{4}\right)_{2} \mathrm{SO}_{4}$, then collecting those precipitated by increasing the concentration to $85 \%$ saturation.

In order to quantify the purification of CSP in this and other steps, it was necessary to demonstrate that, for each bioassay, CSP was limiting and that the amount of Pchlide formed was proportional to the amount of CSP protein added. Fig. 4 shows a titration of CSP from both an unfractionated supernatant sample and a $60-85 \%$-satd.- $\left(\mathrm{NH}_{4}\right)_{2} \mathrm{SO}_{4}$ fraction. Each sample was bioassayed with a constant amount of pellet, which was sufficient to saturate the assay in the presence of the highest CSP concentrations used as determined by a separate experiment. A linear relationship between $\mu \mathrm{g}$ of CSP protein added and cyclase

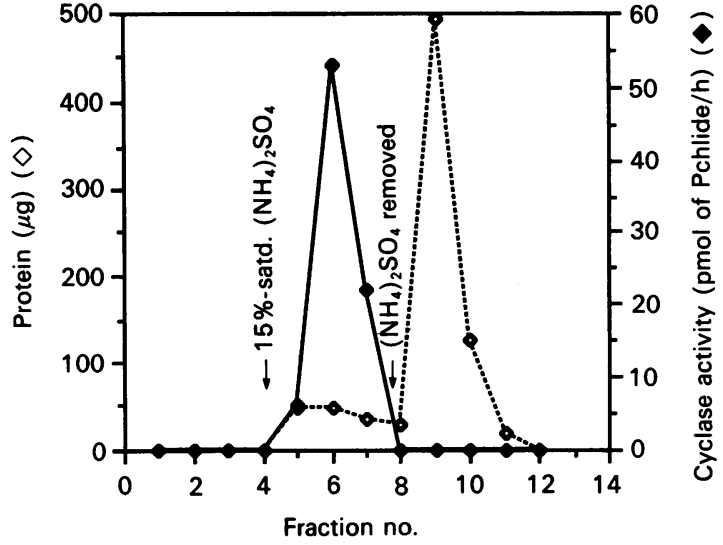

Fig. 5. Chromatography of CSP on phenyl-Sepharose

Column chromatography and fraction bioassay were as described under 'Methods'. A sample of the $60-85 \%$-satd.- $\left(\mathrm{NH}_{4}\right)_{2} \mathrm{SO}_{4}$ fraction $(0.8 \mathrm{mg}$ of protein) was resuspended in $1.5 \mathrm{ml}$ of Buffer $\mathrm{B} / 100 \%$-satd. $\left(\mathrm{NH}_{4}\right)_{2} \mathrm{SO}_{4}(3: 1, \mathrm{v} / \mathrm{v})$ and applied to the column; which had been equilibrated in the same mixture. CSP was eluted by reducing the concentration of $\left(\mathrm{NH}_{4}\right)_{2} \mathrm{SO}_{4}$ in the running buffer to $15 \%$ saturation. The amount of pellet protein per assay was $0.6 \mathrm{mg}$. Substrates and cofactors were as in Table 1. The reduction of $\left(\mathrm{NH}_{4}\right)_{2} \mathrm{SO}_{4}$ saturation to $15 \%$ and the omission of $\left(\mathrm{NH}_{4}\right)_{2} \mathrm{SO}_{4}$ from the running buffer are indicated on the Figure.

activity was observed for both samples. As was expected, the more purified $\left(\mathrm{NH}_{4}\right)_{2} \mathrm{SO}_{4}$ fraction gave a line with a steeper slope than the uncut supernatant. An 8.5-fold purification for the $\left(\mathrm{NH}_{4}\right)_{2} \mathrm{SO}_{4}$-fractionation step was calculated from the ratio of the gradients of these two lines.

Hydrophobic-interaction chromatography. Further purification of CSP after the $\left(\mathrm{NH}_{4}\right)_{2} \mathrm{SO}_{4}$ fractionation was possible by using hydrophobic-interaction chromatography. The behaviour of CSP on a phenyl-Sepharose column is shown in Fig. 5. The sample was loaded in Buffer $\mathbf{B}, 25 \%$ saturated with respect to $\left(\mathrm{NH}_{4}\right)_{2} \mathrm{SO}_{4}$, to promote binding to the column; in the absence of $\left(\mathrm{NH}_{4}\right)_{2} \mathrm{SO}_{4}$ no CSP would bind (results not shown). Decreasing the saturation of $\left(\mathrm{NH}_{4}\right)_{2} \mathrm{SO}_{4}$ in the running buffer to $15 \%$ released some protein, along with the bound CSP. No additional CSP was eluted when $\left(\mathrm{NH}_{4}\right)_{2} \mathrm{SO}_{4}$ was totally removed from the running buffer, but most of the protein $(80 \%)$ was eluted at that point. This column gave a 4.8 -fold purification of CSP with a $35 \%$ recovery of activity.

Fig. 6 shows an SDS/PAGE analysis of the partial purification of CSP from unfractionated supernatant (track 1). Track 2 shows the supernatant after the $60-85 \%$-satd.- $\left(\mathrm{NH}_{4}\right)_{2} \mathrm{SO}_{4}$ fractionation. Many of the supernatant proteins were removed by this step; the major protein band, of molecular mass $52 \mathrm{kDa}$ (track 1), probably corresponds to the large subunit of ribulose bisphosphate carboxylase. Track 3 shows the 60-85\%-satd.$\left(\mathrm{NH}_{4}\right)_{2} \mathrm{SO}_{4}$ fraction after passing through a phenyl-Sepharose column. At this point, four major bands, or groups of bands, were visible on the gel: two single bands of 68 and $47 \mathrm{kDa}$; a group of four proteins ranging from 34 to $38 \mathrm{kDa}$; a doublet of 25 and $27 \mathrm{kDa}$. A few minor proteins were also visible. Although CSP did not bind to Blue Sepharose (Table 4), a small degree of purification was achieved by passing the purified fraction (track 3 ) through this column (track 4). The four proteins of 34-38 kDa were absorbed on to Blue Sepharose and could be eluted by $0.1-0.3 \mathrm{M}-\mathrm{NaCl}$ (results not shown).

Table 5 summarizes the purification data for the various steps described here. The data are expressed as "relative specific activity' since the term 'specific activity' would be misleading in a bioassay system where the addition of another, as yet undefined, 


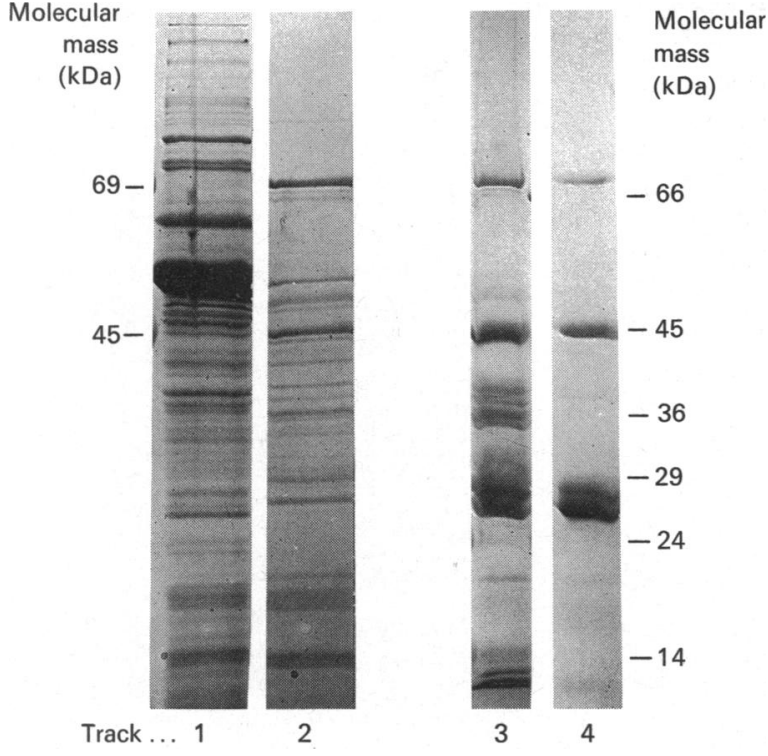

Fig. 6. SDS/PAGE analysis of $\left(\mathrm{NH}_{4}\right)_{2} \mathrm{SO}_{4}$-fractionated, phenylSepharose-purified and Blue-Sepharose-purified supernatant fractions

The Figure shows SDS/PAGE analysis of the supernatant fraction (track 1) after sequential purification by $60-85 \%$ saturation with $\left(\mathrm{NH}_{4}\right)_{2} \mathrm{SO}_{4}$ (track 2), phenyl-Sepharose chromatography (track 3) and Blue Sepharose chromatography (track 4). The molecular-mass values shown are derived from the position of protein standards (see under 'Methods').

\section{Table 5. Summary of the purification of CSP}

Purification factors were calculated as described in the Results section. Fig. 4 displays the rationale of this calculation. $\left(\mathrm{NH}_{4}\right)_{2} \mathrm{SO}_{4}$ fractionation, phenyl-Sepharose and Blue Sepharose chromatography are shown in series starting from unfractionated supernatant (relative specific activity 1). Purification on $\mathrm{MgProtoMe}_{2}-$ Sepharose is shown as a separate experiment.

\begin{tabular}{|c|c|c|}
\hline Purification step & $\begin{array}{l}\text { Relative specific } \\
\text { cyclase activity }\end{array}$ & $\begin{array}{l}\text { Recovery of } \\
\text { cyclase activity } \\
(\%)\end{array}$ \\
\hline$\left(\mathrm{NH}_{4}\right)_{2} \mathrm{SO}_{4}$ fractionation & & \\
\hline Supernatant & 1 & 100 \\
\hline $\begin{array}{l}\text { 60-85\%-satd. }\left(\mathrm{NH}_{4}\right)_{2} \mathrm{SO}_{4} \\
\text { Phenyl-Sepharose }\end{array}$ & 8.5 & 31 \\
\hline $\begin{array}{l}15 \% \text {-satd. }\left(\mathrm{NH}_{4}\right)_{2} \mathrm{SO}_{4} \\
\text { fraction }\end{array}$ & 40.8 & 11 \\
\hline $\begin{array}{l}\text { Blue Sepharose } \\
\text { Unabsorbed fraction }\end{array}$ & 43.4 & 5 \\
\hline $\begin{array}{l}\text { MgProtoMe }_{2}-\text { Sepharose } \\
\text { Supernatant } \\
\text { Unbound } \\
\text { pH 9.7-eluted }\end{array}$ & $\begin{array}{l}1 \\
0.5 \\
9\end{array}$ & $\begin{array}{r}100 \\
21 \\
34\end{array}$ \\
\hline
\end{tabular}

protein fraction is required to measure the enzymic activity. The Table shows that the purification of CSP from the original supernatant fraction achieved by combining $\left(\mathrm{NH}_{4}\right)_{2} \mathrm{SO}_{4}$ fractionation with phenyl-Sepharose and Blue Sepharose chromatography was 43.4-fold; the recovery of CSP was $5 \%$. Clearly, the total purification from the intact plastid, before fractionation into pellet and supernatant, was greater. The affinitychromatography step (Fig. 2), which by itself gave a 9-fold purification of CSP (Table 5) with a $34 \%$ recovery, is essentially an analytical tool which, at the present time, is hardly suitable for preparative use. Therefore it has not yet been combined with the other steps in an overall purification scheme.

\section{DISCUSSION}

Since Wong \& Castelfranco (1984) reported the resolution of the cyclase into soluble and membrane-bound components, no attempt has been made to assign specific roles for these fractions in the cyclization process. Our recent findings (Walker et al., $1988,1989)$ require at least one oxygen-binding protein responsible for the stereospecific hydroxylation of the 6methylpropionate side chain of MgProtoMe, followed by two enzyme components catalysing the formation of the 6- $\beta$ oxopropionate derivative and the completion of cyclization $(C-C$ bond-formation). Therefore we would expect a minimum of three enzymes to be involved in this process.

In the method used here to resolve the cyclase, the use of lengthy ultracentrifugation to separate pellet and supernatant fractions is avoided; hence, the preparation time is greatly reduced compared with the previously used method (Wong \& Castelfranco, 1984). This is an advantage, since the pellet has low stability at $4^{\circ} \mathrm{C}$.

It has been demonstrated that MT activity is contained mainly in the pellet, and negligible activity is associated with CSP. Since reconstitution of the cyclase system can be achieved with MgProtoMe even in the absence of SAM, the possibility of the reconstitution merely involving the addition of a solubilized MT component can be eliminated. H.p.l.c. analysis of the experiments summarized in Table 2 also revealed that CSP contained essentially all the ME activity. This activity has not yet been fully characterized.

At least one of the components of the cyclase has a heavymetal requirement. Chereskin \& Castelfranco (1982) were able to demonstrate an iron requirement in vivo, but saw only a slight inhibition by $\alpha, \alpha^{\prime}$-dipyridyl on Pchlide synthesis in vitro. However, Nasrulhaq-Boyce et al. (1987) reported that, in intact wheat (Triticum aestivum) plastids, 8-hydroxyquinoline inhibited the cyclase, but the more water-soluble desferrioxamine was only slightly inhibitory; these authors suggested that the metal was located in a hydrophobic environment. Our own data (Table 3) confirm this pattern of inhibition. Pretreatment of the pellet fraction with chelator inhibited cyclization, suggesting that a heavy-metal-containing component is embedded in the membrane (Table 3). On the other hand, the metal-ion requirement of the CSP seems to be complex. Inhibition by 8-hydroxyquinoline and desferal mesylate could not be investigated, since removal of these chelators after the CSP treatment was unsuccessful. Using an alternative approach, we have recently demonstrated that the reconstituted cyclase system can be stimulated by $\mathrm{Mg}^{2+}$ ions and that this metal-ion requirement seems to be associated with the CSP (B. J. Whyte, P. Vijayan and P. A. Castelfranco, unpublished work). Other bivalent cations $\left(\mathrm{Ca}^{2+}, \mathrm{Fe}^{2+}, \mathrm{Mn}^{2+}, \mathrm{Cu}^{2+}\right.$ and $\mathrm{Zn}^{2+}$ ) and $\mathrm{La}^{3+}$ are inhibitory, whereas $\mathrm{Na}^{+}$at higher concentration can partially replace $\mathbf{M g}^{2+}$.

The inability of CSP to bind to Blue Sepharose (Table 4) suggests that CSP is not the NADPH-binding component of the cyclase; this raises the possibility that the NADPH-binding protein is located in the membrane. Conceivably, the metal-ion and NADPH-binding functions might both be involved in a membrane-bound oxygen-binding complex as seen, for example, in some mixed-function oxidases. However, to date there is no evidence to suggest the involvement of a haemoprotein in the cyclase.

The CSP had an affinity for $\mathrm{MgProtoMe}_{2}-$ Sepharose (Fig. 2). Although $\mathrm{MgProtoMe}_{2}$ was the porphyrin initially coupled to 
Sepharose, it should be noted that the extracts applied to the column contained ME activity (Chereskin et al. 1982; Wong et al., 1985; Walker et al., 1988); thus this column could have a mixture of MgProto, MgProtoMe and $\mathrm{MgProtoMe}_{2}$ bound to the Sepharose lattice. Elution of CSP with porphyrin solutions was not possible, owing to the high affinity of this gel for such compounds (Richards et al., 1987). The elution of porphyrinbinding proteins from such affinity columns by raising the $\mathrm{pH}$ has been previously reported (Hinchigeri et al., 1981); this was also observed in the present study. Since NADPH was not required to elute CSP from the column, we surmise that NADPH is not needed to bind the porphyrin substrate to CSP. Moreover, the cyclase has a high $\mathrm{pH}$ optimum (pH 9.0, Fig. 1). If the release of the porphyrin from the CSP were the rate-limiting step in this catalysis [as it is with Pchlide reductase (Griffiths 1978)], there might be an interesting correlation between the release of CSP from the affinity column and the release of product from the enzyme in the catalytic sequence.

Reasonable purification of CSP was achieved with a combination of $\left(\mathrm{NH}_{4}\right)_{2} \mathrm{SO}_{4}$ fractionation, phenyl- and Blue Sepharose chromatography (Table 5). Although only four major bands were visible after this purification procedure (Fig. 6), it is still possible that CSP is a minor protein component.

In order to understand the mechanism of the cyclization process, we need to consider tentatively two hypothetical models. The first model would involve a membrane component consisting of an NADPH-dependent oxygen-binding hydroxylating enzyme with the CSP containing all the other proteins required for the cyclization. In the other model, the membrane component would contain all the enzymes necessary for cyclization, and the CSP would act as a $\mathrm{Mg}$-porphyrin carrier. Such a putative porphyrin carrier has recently been proposed in Rhodobacter capsulatus (Bauer \& Marrs, 1988; Bauer et al., 1988).

This research was sponsored by the National Science Foundation grant DCB-8805668. We are indebted to Harris Moran Seeds of Salinas,
CA, U.S.A., for generously providing the cucumber seeds. Dr. K. M. Smith, Dr. P. Vijayan and Dr. J. D. Weinstein read the manuscript before its submission and made many useful suggestions.

\section{REFERENCES}

Bauer, C. E. \& Marrs, B. L. (1988) Proc. Natl. Acad. Sci. U.S.A. 85, 7074-7078

Bauer, C. E., Young, D. A. \& Marrs, B. L. (1988) J. Biol. Chem. 263, 4820-4827

Bradford, M. (1976) Anal. Biochem. 72, 248-254

Castelfranco, P. A., Weinstein, J. D., Schwarcz, S., Pardo, A. T. \& Wezelman, B. E. (1979) Arch. Biochem. Biophys. 192, 592-598

Chereskin, B. M. \& Castelfranco, P. A. (1982) Plant Physiol. 69, 112-116

Chereskin, B. M., Wong, Y.-S. \& Castelfranco, P. A. (1982) Plant Physiol. 70, 987-993

Chereskin, B. M., Castelfranco, P. A., Dallas, J. L. \& Straub, K. M. (1983) Arch. Biochem. Biophys. 226, 10-18

Fuesler, T. P., Castelfranco, P. A. \& Wong, Y-S (1984) Plant Physiol. 74, 928-933

Fuesler, T. P., Hanamoto, C. M. \& Castelfranco, P. A. (1982) Plant Physiol. 69, 421-423

Granick, S. (1950) Harvey Lect. 44, 220-245

Griffiths, W. T. (1978) Biochem. J. 174, 681-692

Hardy, S. I., Castelfranco, P. A. \& Rebeiz, C. A. (1970) Plant Physiol. 46, 705-707

Heyns, N. \& DeMoor, P. (1974) Biochim. Biophys. Acta 358, 1-13

Hinchigeri, S. B., Chan, J. C.-S. \& Richards, W. R. (1981) Photosynthetica 15, 351-359

Laemmli, U. K. (1970) Nature (London) 227, 680-685

Nasrulhaq-Boyce, A., Griffiths, W. T. \& Jones, O. T. G. (1987) Biochem. J. 243, 23-29

Richards, W. R., Walker, C. J. \& Griffiths, W. T. (1987) Photosynthetica 21, 462-471

Walker, C. J., Mansfield, K. E., Rezzano, I. N., Smith, K. M. \& Castelfranco, P. A. (1988) Biochem. J. 255, 685-692

Walker, C. J., Mansfield, K. E., Smith, K. M. \& Castelfranco, P. A. (1989) Biochem. J. 257, 599-602

Wong, Y.-S. \& Castelfranco, P. A. (1984) Plant Physiol. 75, 658-661

Wong, Y.-S. \& Castelfranco, P. A. (1985) Plant Physiol. 79, 730-733

Wong, Y.-S., Castelfranco, P. A., Goff, D. A. \& Smith, K. M. (1985) Plant Physiol. 79, 725-729

Received 20 November/28 January 1991; accepted 4 February 1991 